\section{Curiously strong lipidoids}

\section{By Tim Fulmer, Senior Writer}

Researchers at Alnylam Pharmaceuticals Inc. and the Massachusetts Institute of Technology have developed a class of lipid-like carriers that are nearly 100 times more efficient at delivering small interfering RNA than previously studied lipid-based carriers. ${ }^{1}$ The parties are now scaling up production of one of the carriers-dubbed lipidoids-and are optimizing it in rodent and nonhuman primate disease models.

If successful, lipidoids could address one of the most significant challenges facing RNAi therapeutics: safely and efficiently delivering the molecules to diseased tissue. ${ }^{2}$ Naked siRNA is the simplest option but has primarily been limited to diseases of the eye, in which direct local delivery is possible. For most other indications, systemic delivery is the only way to go, requiring a carrier to package and protect the molecules from serum nucleases and to selectively target the compounds to the desired tissue.

Lipidoids and lipids share many of the physicochemical properties that drive the formation of liposomes for RNAi delivery. However, lipidoids require many fewer steps to synthesize and purify. Those advantages make high throughput combinatorial synthesis of lipidoids and rapid in vitro screening of thousands of potential drug delivery candidates possible.

The Alnylam-MIT team previously evaluated a library of more than 1,200 lipidoids, the best of which delivered siRNA in three different animal models: mice, rats and nonhuman primates. ${ }^{3,4}$ However, the first-generation lipidoids had efficacies that were only comparable to those for existing stable nucleic acid lipid particle formulations, thus requiring siRNA loads in the $\mathrm{mg} / \mathrm{kg}$ body weight range.

The team hoped to improve the therapeutic index and reduce the off-target toxicity of the siRNA loads by lowering dosing to the $\mu \mathrm{g} / \mathrm{kg}$ range, and they produced a second-generation library of 126 epoxidederived lipidoids.

A cell-based screen of the library identified 12 lipidoids that delivered sufficient siRNA to reduce luciferase gene expression by at least $80 \%$ in a transfected cell line. In mouse livers, one of the lipidoids-C12-200-delivered 3, 10 and $30 \mu \mathrm{g} / \mathrm{kg}$ of an siRNA against Factor VII that reduced gene expression by about $25 \%, 50 \%$ and $80 \%$, respectively.

Most lipid-based delivery carriers require siRNA doses of at least $1 \mathrm{mg} / \mathrm{kg}$ to get more than $50 \%$ gene silencing.
"The lipidoid-siRNA formulation described in the PNAS paper showed an incredibly high efficacy, perhaps two or even three orders of magnitude higher than most previously reported lipid-based RNAi delivery formulations," said Niren Murthy, associate professor of biomedical engineering at the Georgia Institute of Technology.

Given the efficient gene silencing by siRNA delivered with C12200 , the researchers hypothesized that the lipidoid might be able to simultaneously deliver multiple siRNA molecules to the liver without generating toxicity.

In mice, a single dose of C12-200 complexed with $0.1 \mathrm{mg} / \mathrm{kg}$ of five different siRNAs reduced expression of all five genes by at least $50 \%$ compared with C12-200 complexed to the same concentration of nontargeted control siRNA. There were no adverse effects in the animals.

Finally, the researchers studied C12-200 in cynomolgus monkeys. An i.v. infusion of C12-200 complexed with 300,100 and $30 \mu \mathrm{g} / \mathrm{kg}$ of siRNA against transthyretin reduced gene expression by about $95 \%, 85 \%$ and $70 \%$ compared with infusion of phosphate control buffer.

The Alnylam-MIT team was led by Daniel Anderson and Robert Langer, researchers in MIT's David H. Koch Institute for Integrative Cancer Research, and included Akin Akinc, associate director of research at Alnylam. The paper was published in the Proceedings of the National Academy of Sciences.

\section{Low rider}

-Akin Akinc,

The potential for low dosing is a key advantage of lipidoids over other RNAi delivery techniques, according to John Rossi, chair of the Department of Molecular Biology at the Beckman Research Institute at City of Hope and cofounder of RNAi biotech Dicerna Pharmaceuticals Inc.

Low doses could translate into improved safety, with "reduced offtarget tissue toxicity and lower intracellular toxicity that can arise from high levels of exogenous siRNA interfering with the endogenous RNAi processing machinery," Rossi said.

To show this, Murthy wants to see more long-term safety data. "While the lipidoid carrier is a strong candidate for a clinical trial given its outstanding efficacy, a key next step is looking at the safety of long-term administration, as would occur when treating any chronic disease or condition," he said. "Weight loss and liver toxicity should be monitored in the animals over several months of lipidoid-siRNA administration."

Murthy and colleagues have inhibited gene expression in the liver at siRNA levels as low as those described in the PNAS paper. ${ }^{5}$ His group used hydrophobic polymers called polyketals as the siRNA delivery vehicle.

He said polyketals are strongly hydrophobic and should have better stability than lipid-based systems.

\section{Beyond the liver}

Charles Roth, associate professor of chemical and biochemical engineering at Rutgers University, said it was still unclear exactly how the lipidoids preferentially localized to the liver parenchyma. He said a detailed pharmacokinetics study should help show how specific the 
liver effect is and the mechanism by which hepatocytes and/or liver macrophages mediate uptake of the lipidoids.

Since the lipidoids are not degradable, it will be important to investigate the possibility of their accumulating over time and whether that has any consequences, Roth added.

Another question is whether C12-200 can deliver siRNA to other tissues as efficiently as to the liver, said Paloma Giangrande, assistant professor of internal medicine and member of the Holden Comprehensive Cancer Center at The University of Iowa.

Giangrande's lab has focused on developing aptamer technology for targeting siRNA to tumors. ${ }^{6}$ Roth's group has focused on two different siRNA delivery approaches-dendrimers and copolymers. ${ }^{7,8}$

The MIT and Alnylam researchers will continue working together to optimize C12-200, including finding the best dosing regimen and scaling up manufacturing, said Anderson.

As part of their work, Akinc said the team plans to package C12200 with a variety of siRNA molecules against targets associated with multiple diseases. "That will help us better understand whether the C12-200-siRNA complex can potentially target genes in tissues and organ types other than the liver," he said.

Alnylam is developing other siRNA delivery strategies in parallel with lipidoids, including lipid nanoparticles and siRNA conjugation strategies.

"The proper choice of an efficient RNAi delivery strategy depends on the tissue and cellular pathway targeted in a particular disease. It's probably unwise to assume a single type of delivery vehicle would have a good therapeutic index in all diseases," said Akinc.

Bob Brown, SVP of research at Dicerna, agreed that flexibility will be key. "The choice of a delivery strategy will depend on the types of RNAi molecule being used, as well as which tissues are targeted in a particular disease," he said.

"For example," said Brown, "while lipid-based nanocarriers are suited for delivering RNAi to solid tumors, they generally aren't suitable for liquid tumors, where RNAi-protein or RNAi-aptamer conjugates would perhaps work better. Moreover, in special cases where local delivery is possible, as in the eye, no delivery vehicle may be needed at all."

Rossi and Dicerna have focused on delivering Dicer substrate RNAi molecules using aptamer technology. ${ }^{9,10}$ To help them develop that strategy, Dicerna is collaborating with the aptamer-focused biotech Archemix Corp.

Multiple patents cover the lipidoids and their use as delivery vehicles to treat multiple diseases, said Anderson. Akinc told SciBX that Alnylam has licensed the IP.

Fulmer, T. SciBX 3(2); doi:10.1038/scibx.2010.34

Published online Jan. 14, 2010

\section{REFERENCES}

1. Love, K. et al. Proc. Natl. Acad. Sci. USA; published online Jan. 11, 2010; doi:10.1073/pnas.0910603106

Contact: Daniel Anderson, Massachusetts Institute of Technology, Cambridge, Mass.

e-mail: dgander@mit.edu

2. Castanotto, D. \& Rossi, J. Nature 457, 426-433 (2009)

3. Akinc, A. et al. Nat. Biotechnol. 26, 561-569 (2008)

4. Akinc, A. et al. Mol. Ther. 17, 872-879 (2009)

5. Lee, S. et al. Nucleic Acids Res. 37, e145 (2009)

6. Dassie, J. et al. Nat. Biotechnol. 27, 839-846 (2009)

7. Waite, C. et al. BMC Biotechnol. 9, 38 (2009)

8. Peddada, L. et al. J. Control Release 140, 134-140 (2009)

9. Zhou, J. et al. Mol. Ther. 16, 1481-1489 (2008)

10. Zhou, J. et al. Nucleic Acids Res. 37, 3094-3109 (2009)

\section{COMPANIES AND INSTITUTIONS MENTIONED}

Alnylam Pharmaceuticals Inc. (NASDAQ:ALNY), Cambridge, Mass. Archemix Corp., Cambridge, Mass.

Beckman Research Institute at City of Hope, Duarte, Calif. Dicerna Pharmaceuticals Inc., Watertown, Mass. Georgia Institute of Technology, Atlanta, Ga. Massachusetts Institute of Technology, Cambridge, Mass. Rutgers University, Piscataway, N.J.

The University of lowa, lowa City, lowa 